\title{
BMJ Open Illness perception in patients with chronic kidney disease and kidney failure: a scoping review protocol
}

\author{
Jia Hwei Ng (D) , ${ }^{1}$ Jaclyn Vialet, ${ }^{2}$ Michael A Diefenbach ${ }^{3}$
}

To cite: $\mathrm{Ng} \mathrm{JH}$, Vialet J, Diefenbach MA. Illness perception in patients with chronic kidney disease and kidney failure: a scoping review protocol. BMJ Open 2021;11:e042298. doi:10.1136/ bmjopen-2020-042298

- Prepublication history and additional material for this paper is available online. To view these files, please visit the journal online (http://dx.doi.org/10. 1136/bmjopen-2020-042298).

Received 30 June 2020 Revised 27 January 2021 Accepted 05 February 2021

Check for updates

(c) Author(s) (or their employer(s)) 2021. Re-use permitted under CC BY-NC. No commercial re-use. See rights and permissions. Published by BMJ.

${ }^{1}$ Division of Kidney Diseases and Hypertension, Donald and Barbara Zucker School of Medicine at Hofstra/Northwell, Great Neck, New York, USA ${ }^{2}$ Clinical Medical Library, North Shore University Hospital, Manhasset, New York, USA

${ }^{3}$ Center of Health Innovation and Outcomes Research, Manhasset, New York, USA

Correspondence to

Dr Jia Hwei Ng;

jng10@northwell.edu

\section{ABSTRACT}

Introduction Patient education is a crucial component in the management of chronic kidney disease (CKD); however, many education programmes do not tailor to patients' disease-related belief and emotional reactions. According to Leventhal's self-regulation model, such beliefs and reactions, known as illness perceptions, are a part of patients' understanding of their disease and treatmentrelated actions. The existing research of illness perception in the field of nephrology has been heterogenous in terms of study outcomes and in study population; and the evidence of how illness perceptions affect behavioural and decisional outcomes is limited. This scoping review aims to map and summarise the evidence of published literature on illness perception among patients with CKD not yet treated by dialysis and kidney failure.

Methods and analysis This study bases its approach on Joanna Brigg's Institute Guidelines on scoping review methods. The search strategy was developed together with a medical information specialist. Searches will be performed on acceptance of publication in the following databases: PubMed, EMBASE, PsycINFO via Ovid, Scopus and the Cumulative Index to Nursing and Allied Health Literature. Searches will be run without incorporating a date restriction in order to capture content from the databases' inception to present day. Search terms including 'illness perception', 'kidney disease' and 'kidney failure' will be screened in titles and abstracts. Two independent researchers will screen the abstracts and full text for full eligibility. We will include studies focusing on illness perception of patients with CKD with estimated glomerular filtration rate $<60 \mathrm{~mL} / \mathrm{min} / 1.73 \mathrm{~m}^{2}$, kidney failure or recipients of kidney transplant. We will exclude patients $<18$ years of age, patients with acute kidney injury and non-English articles. All demographic data, study design and study findings will be collected and analysed using a data abstraction tool.

Ethics and dissemination This study does not require internal review board approval. We will present the findings of this scoping review in a peer-reviewed journal.

\section{INTRODUCTION}

Chronic kidney disease (CKD) affects 9\% of the population worldwide, with 2.5 million people currently receiving treatment for kidney failure every year. ${ }^{1}$ Kidney failure is associated with greater disease burden, higher rates of hospitalisation and higher

\section{Strengths and limitations of this study}

- This will be the first scoping review to identify literature on illness perception according to the selfregulation model among patients with both chronic kidney disease and kidney failure.

- The search strategy includes the use of an established scoping review methodology.

- We have included a medical information specialist in the main research team.

- The search strategy may not be sensitive to studies that included a heterogeneous population of people with chronic diseases.

- The findings will be limited to articles written in English.

mortality. ${ }^{2}$ In order to optimise the management of kidney disease and to slow progression of disease, guidelines from the WHO and several national kidney societies have recommended patient education and selfmanagement programmes. $^{3-6}$ Despite the increase in education and self-management programmes, the effectiveness of these programmes in changing behaviour and outcomes has been inconsistent. ${ }^{7}$ One of the challenges with existing programmes is the lack of targeting the intervention to patients' specific cultural expectations or tailoring its content to individual disease-related beliefs, expectations and emotional reactions. ${ }^{8-10}$

Individuals with acute or chronic diseases form their own belief systems to make sense of and cope with their medical condition. ${ }^{112}$ Such belief systems, also known as illness perceptions are theorised within Leventhal's Common Sense Model of self-regulation. Illness perceptions are defined by the following attributes: identity (what the disease is), cause (what caused it), timeline (acute, chronic, cyclic), consequences (minor, moderate, severe), control (whether anything can be done about the disease) and emotional response (anxiety, worry). ${ }^{11}{ }^{12}$ Research on illness perceptions has shown that improved adherence to recommended regimens and 
improved health outcomes are associated with medically accurate illness perceptions. Furthermore, studies across a number of medical subspecialties (eg, cardiology, endocrinology and rehabilitation) have shown that individuals' illness perceptions are amenable to interventions. ${ }^{13-16}$ However, the evidence on illness perceptions in the domain of kidney health is less well established.

\section{STUDY RATIONALE}

In the field of nephrology, the existing work on illness perception has been heterogenous and wide ranging in terms of study outcomes and study population. ${ }^{17-20}$ Studies have shown that patients who hold positive illness perceptions about their kidney disease have better psychosocial and clinical outcomes, specifically, greater quality of life, treatment adherence and survival. ${ }^{18}{ }^{21-24}$ Additionally, illness perceptions vary across CKD at different categories of estimated glomerular filtration rate (eGFR) and treatment modalities in response to kidney failure. ${ }^{25-28}$ Patients receiving dialysis perceive illness consequences as more severe than those who have not yet started dialysis. Further, once patients have started dialysis, they are more likely to consider their illness as chronic versus acute. ${ }^{25}$ When it comes to kidney failure treatment modalities, patients on home-based dialysis indicate that they have higher treatment control compared with patients on in-centre haemodialysis. ${ }^{26}{ }^{27}$ While illness perception is an emerging area of research in nephrology, the majority of studies are cross-sectional in design, with only a few prospective observational studies and randomised-controlled trials. ${ }^{21-24} 2829$ Moreover, the effect of illness perception on behavioural/ decisional outcomes such as self-management or decisionmaking has not been studied. Given the heterogeneity of prior research studies in this topic, a scoping review allows us to examine the breadth of the studies, identify available evidence, analyse knowledge gaps and identify new developments in the field.

\section{STUDY AIMS}

This aim of this study is to explore the extent and nature of published literature on illness perception according to the self-regulation model, map and summarise the evidence, and inform future research among patients across the different stages of CKD and kidney failure.

Through discussions with the research team, the research questions are defined as:

1. What are the differences in illness perception across the different categories of CKD by eGFR and kidney failure?

Does illness perception change with time?

2. What are the differences in illness perceptions for patients with kidney failure receiving different types of dialysis including haemodialysis and peritoneal dialysis?

3. To what extent do illness perceptions affect clinical, psychosocial, behavioural and decisional outcomes?
Clinical outcomes include CKD progression, medication adherence, dialysis treatment adherence and mortality.

Psychosocial and behavioural outcomes include depression, quality of life, anxiety, self-control, selfmanagement, return to work and physical activity.

Decisional outcomes include treatment related shared decision-making, decisional regret, and decisional conflict.

4. Which interventions incorporating illness perceptions have been investigated or being developed?

What are the reported effects and outcomes from such interventions?

\section{METHODS}

\section{Protocol development}

The protocol was developed according to the Joanna Brigg's Institute Guidelines. ${ }^{30}$ In order to ensure clear and transparent reporting of the data, the results of the search and the study inclusion process will be reported in full in the final scoping review and presented in a Preferred Reporting Items for Systematic Reviews and Meta-Analyses (PRISMA) extension for scoping review flow diagram. ${ }^{31}$

\section{Study definitions}

$\mathrm{CKD}^{32}$-patients who have eGFR $<60 \mathrm{~mL} / \mathrm{min} / 1.73 \mathrm{~m}^{2}$ for a minimum of 3 months and not yet treated by dialysis. CKD is further categorised into eGFR categories: G3a, eGFR $45-90 \mathrm{~mL} / \mathrm{min} / 1.73 \mathrm{~m}^{2}$; G3b, eGFR $30-44 \mathrm{~mL} /$ $\mathrm{min} / 1.73 \mathrm{~m}^{2} ; \mathrm{G} 4$, eGFR $15-29 \mathrm{~mL} / \mathrm{min} / 1.73 \mathrm{~m}^{2}$.

Kidney failure ${ }^{32}$-patients who have eGFR $<15 \mathrm{~mL} /$ $\min / 1.73 \mathrm{~m}^{2}$ for a minimum of 3 months and or treated with maintenance dialysis

Kidney transplantation-recipient of a kidney transplant.

Treatment modality - the form of treatment when patient goes into kidney failure. This includes haemodialysis, peritoneal dialysis and conservative care management.

\section{Search strategy}

The following databases will be searched from inception onwards on acceptance of this protocol: PubMed (MEDLINE), EMBASE, Scopus, PsycINFO and the Cumulative Index to Nursing and Allied Health Literature. The literature search will be supplemented by scanning the reference lists of included studies and searching grey literature sources, such as Google Scholar, as well as conference proceedings and abstracts published by select journals and organisations, including but not limited to the International Society of Nephrology, World Congress of Nephrology, American Society of Nephrology and the National Kidney Foundation.

A preliminary search was conducted in PubMed on 24 April 2020 to identify, broadly, the varying types of studies available which assess the impact of CKD, kidney failure and kidney transplantation on emotional response, clinical outcomes and decision making. Search terms were analysed and determined for use based on these results and from input provided by the research team and 
information specialist. A draft of the search strategy, which can be found in the online supplemental material 1 , is comprised of Medical Subject Headings and free text terms. The search strategy will be modified as necessary for the other databases. The search strategy will not be limited by study design, year or language of dissemination. The final search strategy will be peer reviewed by another information specialist using the Peer Review of Electronic Search Strategies checklist. ${ }^{33}$

\section{Eligibility criteria}

The proposed scoping review will include works published in peer-reviewed academic journals, doctoral dissertations, research reports and conference abstracts. The type of study designs that are eligible include the following: meta-analysis, systematic reviews, scoping reviews, narrative reviews; randomised-controlled trials, quasi-experimental studies; observational, qualitative studies; letter to the editors; and published protocols with published results of the following: systematic reviews, scoping reviews, clinical trials and cohort study registries. Non-English published work will be excluded, as translation services are not available for this study.

The eligibility criteria of studies are based on the population, concept, context framework.

Population-this refers to: (1) patients with CKD not yet receiving kidney replacement therapy and (2) patients with kidney failure on different kidney replacement therapy (haemodialysis, home haemodialysis, peritoneal dialysis or conservative management), (3) patients with kidney transplantation. Pregnant patients will be included. There will be no limit to patients' country origin or health system. Studies involving non-human subjects will be not be eligible. Studies involving patients $<18$ years of age will be excluded.

Studies involving patients with acute kidney injury will be excluded. Articles not written in English will also be excluded.

Concept-we will investigate the concept of illness perception or illness representation, a theoretical mental framework based on the Common-Sense Model of selfregulation. ${ }^{11} 12$

Context-we will include studies in the outpatient and inpatient healthcare setting, as well as community setting.

\section{Study selection}

Titles and abstracts of articles will be independently reviewed by two authors (JHN and MD). JHN is a nephrologist and clinical epidemiologist, and MD is a behavioural scientist who is an expert in the self-regulation model. If the articles are representative of the inclusion criteria, the articles will go through two full-text independent reviews by two authors (JHN and MD). In order to ensure interrater reliability, both the reviewers will assess $20 \%$ of the articles independently at both the title and abstract review stage and at the full article review stage. If disagreements arise either at the abstract or full text phase of the review, the two reviewers will discuss until consensus is reached. If consensus cannot be reached, a third-party reviewer would be consulted. A draft of the PRISMA inclusion and exclusion flow chart is included in the online supplemental material 1.

\section{Data extraction}

A data charting form was developed by the team to collect information on the following:

1. Article identifiers (authors, year of publication, objective).

2. Study identifiers (sample size, design, country, length of follow-up, inclusion and exclusion criteria).

3. Setting and population (age, sex, gender, CKD with eGFR $<60 \mathrm{~mL} / \mathrm{min} / 1.73 \mathrm{~m}^{2}$, kidney failure, kidney transplantation, type of kidney replacement modality, inpatient/outpatient/community setting).

4. Comorbid conditions (diabetes, hypertension, cardiovascular disease, cancer, HIV, etc.).

5. How illness perception is applied in the study (as the exposure or outcome).

Domains of illness perception assessed (identity, cause, timeline, consequences, control, emotional representation).

6. Validated instruments used for illness perception, if applicable, such as Illness Perception Questionnaire (IPQ), Revised IPQ and Brief IPQ. ${ }^{30-32}$

7. Themes related to the domains of illness perception (for qualitative studies).

8. Outcome measures (clinical, psychosocial/behavioural and decisional outcomes).

If a particular data field is not available, we will attempt to contact the authors to obtain more information.

\section{Collating, summarising and reporting of results}

We will perform descriptive summary statistics to reflect the number of studies included, study design, CKD at different eGFR categories, kidney failure, kidney transplantation, characteristics of study population, exposures and outcomes, publication year and country where studies were conducted. The data will be presented in the form of tables and figures. For qualitative studies, we will summarise the themes provided by the authors. The draft of the data charting form is provided in the online supplemental material 1 .

\section{Limitations}

There are several limitations to the study. The search strategy may not be sensitive to studies that included a heterogeneous population of people with chronic diseases (including some with kidney disease). Although we are using five large databases in the search strategy, we may miss relevant publications outside of these databases. We will also miss non-English publications due to the restriction in English language articles.

\section{Patient and public involvement}

Patients and the public were not involved in the development of this protocol. 


\section{ETHICS AND DISSEMINATION}

As the scoping review methodology involves abstracting data from publicly available sources, this study does not require internal review board approval. To the best of our knowledge, this scoping review is the first to map and summarise the literature on illness perception across the CKD at different eGFR categories, kidney failure and kidney transplantation. The results from this scoping review will identify the knowledge gaps and guide the next phase of research in this area. We will present the findings of this scoping review at national conferences and publish the results in a peer-reviewed journal.

\section{Twitter Jia Hwei Ng @jiahweing}

Contributors JHN led the design and conceptualisation of this work and drafted the protocol. JHN, JV and MD were involved in the conceptualisation of the review design, specifically in establishing the inclusion and exclusion criteria. JHN drafted the background section of the protocol. JV and JHN drafted the methodology section of the manuscript. JV developed the search strategy, conducted the search, provided feedback on the manuscript and copyedited the manuscript. MD provided guidance to the conceptualisation and design of the study and data analyses and has revised all drafts of this manuscript for important intellectual content and clarity. All authors give approval to the publishing of this protocol manuscript.

Funding This work was supported by grants $1 \mathrm{U} 010 \mathrm{H} 011690-01-00$ by the Centers of Disease Control and 1R01CA224918-01A1 by the National Cancer Institute, and a Research Scholar Award by the American Cancer Society RSG-15021-01-CPPB to MD.

Competing interests None declared.

Patient consent for publication Not required.

Provenance and peer review Not commissioned; externally peer reviewed.

Supplemental material This content has been supplied by the author(s). It has not been vetted by BMJ Publishing Group Limited (BMJ) and may not have been peer-reviewed. Any opinions or recommendations discussed are solely those of the author(s) and are not endorsed by BMJ. BMJ disclaims all liability and responsibility arising from any reliance placed on the content. Where the content includes any translated material, BMJ does not warrant the accuracy and reliability of the translations (including but not limited to local regulations, clinical guidelines, terminology, drug names and drug dosages), and is not responsible for any error and/or omissions arising from translation and adaptation or otherwise.

Open access This is an open access article distributed in accordance with the Creative Commons Attribution Non Commercial (CC BY-NC 4.0) license, which permits others to distribute, remix, adapt, build upon this work non-commercially, and license their derivative works on different terms, provided the original work is properly cited, appropriate credit is given, any changes made indicated, and the use is non-commercial. See: http://creativecommons.org/licenses/by-nc/4.0/.

\section{ORCID iD}

Jia Hwei Ng http://orcid.org/0000-0002-2276-4111

\section{REFERENCES}

1 Bikbov B, Purcell CA, Levey AS, et al. Global, regional, and national burden of chronic kidney disease, 1990-2017: a systematic analysis for the global burden of disease study 2017 . The Lancet 2020;395:709-33.

2 Saran R, Robinson B, Abbott KC, et al. Us renal data system 2018 annual data report: epidemiology of kidney disease in the United States. Am J Kidney Dis 2019;73:A7-8.

3 Levin A, Stevens PE. Summary of KDIGO 2012 CKD guideline: behind the scenes, need for guidance, and a framework for moving forward. Kidney Int 2014;85:49-61.

4 Narva AS, Norton JM, Boulware LE. Educating patients about CKD: the path to self-management and patient-centered care. Clin J Am Soc Nephrol 2016;11:694-703.
5 Luyckx VA, Tonelli M, Stanifer JW. The global burden of kidney disease and the sustainable development goals. Bull World Health Organ 2018;96:414-22.

6 Li PK-T, Garcia-Garcia G, Lui S-F, et al. Kidney health for everyone everywhere - from prevention to detection and equitable access to care. Blood Purif 2021;50:1-8.

7 Lopez-Vargas PA, Tong A, Howell M, et al. Educational Interventions for patients With CKD: A Systematic Review. Am J Kidney Dis 2016;68:353-70.

8 Kreuter MW, Lukwago SN, Bucholtz RDDC, et al. Achieving cultural appropriateness in health promotion programs: targeted and tailored approaches. Health Educ Behav 2003;30:133-46.

9 Perez A, Acculturation PA. Acculturation, health literacy, and illness perceptions of hypertension among Hispanic adults. J Transcult Nurs 2015;26:386-94.

10 Waterman AD, Browne T, Waterman BM, et al. Attitudes and behaviors of African Americans regarding early detection of kidney disease. Am J Kidney Dis 2008;51:554-62.

11 Diefenbach MA, Leventhal $\mathrm{H}$. The Common-Sense model of illness representation: theoretical and practical considerations. J Soc Distress Homeless 1996;5:11-38.

12 Leventhal H, Phillips LA, Burns E. The Common-Sense model of self-regulation (CSM): a dynamic framework for understanding illness self-management. J Behav Med 2016;39:935-46.

13 Kale MS, Federman AD, Krauskopf K, et al. The association of health literacy with illness and medication beliefs among patients with chronic obstructive pulmonary disease. PLoS One 2015;10:e012 3937-e37.

14 Keogh KM, White P, Smith SM, et al. Changing illness perceptions in patients with poorly controlled type 2 diabetes, a randomised controlled trial of a family-based intervention: protocol and pilot study. BMC Fam Pract 2007;8:36.

15 Petrie KJ, Cameron LD, Ellis CJ, et al. Changing illness perceptions after myocardial infarction: an early intervention randomized controlled trial. Psychosom Med 2002;64:580-6.

16 van der Have M, Brakenhoff LKPM, van Erp SJH, et al. Back/joint pain, illness perceptions and coping are important predictors of quality of life and work productivity in patients with inflammatory bowel disease: a 12-month longitudinal study. J Crohns Colitis 2015;9:276-83.

17 Fowler C, Baas LS. Illness representations in patients with chronic kidney disease on maintenance hemodialysis. Nephrol Nurs $J$ 2006;33:173-4, 179-86-173-4, 79-86.

18 Knowles S, Swan L, Salzberg M, et al. Exploring the relationships between health status, illness perceptions, coping strategies and psychological morbidity in a chronic kidney disease cohort. Am J Med Sci 2014;348:271-6.

19 Knowles SR, Castle DJ, Biscan SM, et al. Relationships between illness perceptions, coping and psychological morbidity in kidney transplants patients. Am J Med Sci 2016;351:233-8.

20 Vélez-Vélez E, Bosch RJ, perception I. Illness perception, coping and adherence to treatment among patients with chronic kidney disease. J Adv Nurs 2016;72:849-63.

21 Chilcot J, Wellsted D, Farrington K. Illness representations are associated with fluid nonadherence among hemodialysis patients. $J$ Psychosom Res 2010;68:203-12.

22 Meuleman Y, Chilcot J, Dekker FW, et al. Health-Related quality of life trajectories during predialysis care and associated illness perceptions. Health Psychol 2017;36:1083-91.

23 Parfeni M, Nistor I, Covic A. A systematic review regarding the association of illness perception and survival among end-stage renal disease patients. Nephrol Dial Transplant 2013;28:2407-14.

24 Alehe S, Kobra P, Azad R. Effect of illness perception promoting interventions on treatment adherence in hemodialysis patients: a randomized controlled trial. Journal of Critical Care Nursing 2013;6:77-86.

25 Jansen DL, Heijmans MJWM, Rijken M, et al. Illness perceptions and treatment perceptions of patients with chronic kidney disease: different phases, different perceptions? Br J Health Psychol 2013;18:244-62.

26 Griva K, Davenport A, Harrison M, et al. An evaluation of illness, treatment perceptions, and depression in hospital- vs. home-based dialysis modalities. J Psychosom Res 2010;69:363-70.

27 Jayanti A, Foden P, Wearden A, et al. Illness beliefs in end stage renal disease and associations with self-care modality choice. PLoS One 2016;11:e0154299.

28 Tasmoc A, Hogas S, Covic A. A longitudinal study on illness perceptions in hemodialysis patients: changes over time. Arch Med Sci 2013;9:831-6.

29 Meuleman Y, de Goeij MCM, Halbesma N, et al. Illness perceptions in patients on predialysis care: associations with time until start 
of dialysis and decline of kidney function. Psychosom Med 2015;77:946-54.

30 Peters MDJ GC, McInerney P, Munn Z. Chapter 11: scoping reviews (2020 version). In: Aromataris E, Munn Z, eds. JBI reviewer's manual. 2020. Adelaide: JBI, 2020

31 Tricco AC, Lillie E, Zarin W, et al. PRISMA extension for scoping reviews (PRISMA-ScR): checklist and explanation. Ann Intern Med 2018;169:467-73.
32 Levey AS, Eckardt K-U, Dorman NM, et al. Nomenclature for kidney function and disease: report of a kidney disease: improving global outcomes (KDIGO) consensus conference. Kidney Int 2020;97:1117-29.

33 McGowan J, Sampson M, Salzwedel DM, et al. PRESS peer review of electronic search strategies: 2015 guideline statement. J Clin Epidemiol 2016;75:40-6. 